\title{
Estructura y composición arbórea del bosque tropical caducifolio secundario en la Depresión Central, Chiapas, México
}

\section{Structure and composition of trees of secondary deciduous dry forest in the Central Depression, Chiapas, Mexico}

\author{
Mercedes Concepción Gordillo Ruiz,2, Miguel Ángel Pérez Farrera³ y Miguel Ángel Castillo Santiago ${ }^{4 *}$ \\ 1 Universidad de Ciencias y Artes de Chiapas. \\ Doctorado en Ciencias en Desarrollo Sustentable. \\ Tuxtla Gutiérrez, Chiapas, México. \\ marip_10@yahoo.com \\ 2 Secretaría de Medio Ambiente e Historia Natural. \\ Departamento de Gestión, Investigación y \\ Educación Ambiental. Tuxtla Gutiérrez, Chiapas, \\ México. \\ 3 Universidad de Ciencias y Artes de Chiapas. * Autor de correspondencia. mcastill@ecosur.mx \\ Facultad de Biología. Herbario Eizi Matuda. Tuxtla \\ Gutiérrez, Chiapas, México. \\ miguel.perez@unicach.mx \\ 4 El Colegio de la Frontera Sur. Departamento de \\ Observación y Estudio de la Tierra, la Atmósfera y e \\ Océano. San Cristobal de las Casas, Chiapas, \\ Mexico.
}

\section{RESUMEN}

El bosque tropical caducifolio está altamente amenazado y transformado a causa de las actividades agrícolas en Chiapas; sin embargo, se conoce poco acerca de su dinámica sucesional y potencial de regeneración. El objetivo de la investigación fue evaluar la capacidad regenerativa de los bosques secundarios a través de los cambios en la riqueza, diversidad, composición y estructura en una reserva forestal de la Depresión Central de Chiapas. Se establecieron 20 unidades de muestreo (UM) de $1000 \mathrm{~m}^{2}$ en bosques con diferentes edades de abandono (C10, C19, C35 y > C40, que representan 10 años, 19 años, 35 años y 40 años, respectivamente), en las que se midieron todos los individuos $\geq 5 \mathrm{~cm}$ de diámetro normal (DN). Se compararon en cada condición atributos de estructura y diversidad mediante análisis de varianza y prueba de comparación de medias Tukey $(p<0.05)$ y la composición florística a través de análisis de ordenación y clasificación. Se registró un total de 142 especies agrupadas en 96 géneros y 41 familias. Leguminosae fue la familia con más especies e individuos. Las especies con mayores abundancias relativas fueron Montanoa tomentosa (5.1\%) y Tecoma stans (5\%). Se encontraron diferencias significativas $(p<0.05)$ en la riqueza acumulada, diversidad (Shannon-Weiner $\mathrm{H}^{\prime}$ y ${ }^{1} \mathrm{D}$ ), densidad de individuos (ind ha- ${ }^{-1}$ ), altura máxima $(\mathrm{m})$, área basal $\left(\mathrm{m}^{2} \mathrm{ha}^{-1}\right)$ y biomasa aérea $\left(\mathrm{Mg} \mathrm{ha}{ }^{-1}\right)$. El procedimiento de análisis de varianza multivariado con permutaciones indicó diferencias significativas $(p<0.05)$ en la composición de especies entre las condiciones tempranas $($ C10 y C19) y las más avanzadas (C35 - C40). Se concluyó que la estructura y composición florística del bosque secundario se recupera lento (baja resilencia), por lo que se requiere implementar actividades conducentes a su conservación en el corto plazo.

PALABRAS CLAVE: comunidad árborea, disturbio, diversidad, similitud florística, sucesión ecologica, resiliencia.

\section{ABSTRACT}

The tropical deciduous forest is threatened and disturbed by agricultural activities; however, their successional dynamics and regeneration potential it is still little known. The objective of this research was assessing the regenerative capacity of the secondary deciduous forests of a Forest Reserve in Chiapas through comparison changes in richness, diversity, composition, and structure indicators. Twenty 1000$\mathrm{m}^{2}$ sampling plots were established in secondary forests with different ages of abandonment (10,19,35 and $>40$ years). All woody plants with diameter at breast height $(\mathrm{DBH}) \geq 5 \mathrm{~cm}$ were measured. Statistics about structure and diversity were calculated in each age-class; ANOVA and Tukey test $(\mathrm{p}<0.05)$ were employed to detect differences between them. Changes in the floristic composition were evaluated through ordination and classification analysis. 142 species grouped into 96 genera and 41 families were recorded. Leguminosae was the family with more species and individuals. The species with the highest relative abundances were Montanoa tomentosa (5.1\%) and Tecoma stans $(5 \%)$. Significative differences were found $(p<0.05)$ in accumulated richness, diversity (Shannon - Weiner $\mathrm{H}^{\prime}$ and 1D), the density of individuals (ind ha-1), maximum height $(\mathrm{m})$, basal area $\left(\mathrm{m}^{2} \mathrm{ha}^{-1}\right)$ and aerial biomass $\left(\mathrm{Mg} \mathrm{ha}^{-1}\right)$. The permutational multivariate analysis of variance showed significative differences $(p<0.05)$ in species composition between early (10 years and 19 years) and more advanced conditions ( 35 and $>40$ years). Our results support the notion that secondary forests recover their composition and structure slowly. Therefore, it is essential to implement activities conducive to its conservation in the short term.

KEYWORDS: tree community, disturbance, diversity, floristic similarity, ecological succession, resilience. 


\section{INTRODUCCIÓN}

El bosque tropical caducifolio (BTC) resguarda una importante diversidad florística y un elevado número de especies endémicas, por lo que es considerado un ecosistema de alto valor ecológico (Rzedowski, 1991). A pesar de su amplia distribución, en México la mayor parte del bosque se encuentra severamente perturbado, aproximadamente $60 \%$ de la distribución original ha sido transformada a terrenos de uso agropecuario y los fragmentos residuales se encuentran severamente amenazados (Trejo y Dirzo, 2000). Su nivel de riesgo en algunas regiones del país es sumamente alto debido a que ocupa suelos fértiles, compitiendo en ese sentido con las actividades agropecuarias. En regiones como el bajío, la parte central de Veracruz y Chiapas, el bosque original prácticamente ha sido eliminado (Pérez-Farrera y Espinoza, 2010; Rzedowski y Calderón, 2013), en estos espacios se encuentran ahora paisajes dominados por usos agrícolas y fragmentos de bosque secundario en diferentes estadios sucesionales. Por lo tanto, el conocimiento sobre el nivel de perturbación y potencial de recuperación de los bosques secundarios es clave para promover estrategias de conservación y restauración del bosque tropical (Quesada et al., 2009; William-Linera et al., 2011).

Tradicionalmente se ha buscado explicar cómo las comunidades vegetales se integran y responden al disturbio a través del estudio de la sucesión ecológica (Guariguata y Ostertag, 2002). Para el caso del BTC, este proceso está menos documentado en comparación con el bosque húmedo (Quesada et al., 2009; Vieria y Scariot, 2006); aunque las evidencias actuales procedentes de modelos determinísticos y estocásticos dan muestra de la alta heterogeneidad en los patrones, mecanismos y trayectorias que siguen las comunidades en recuperación del BTC (Lebrija-Trejos, Meave, Poorter, Pérez-García y Bongers, 2010; Villalobos, 2012; Norden et al., 2015). Al mismo tiempo, se ha comenzado a evaluar la resiliencia del BTC a través del análisis del proceso sucesional (Lebrija-Trejos, Bongers, Pérez-García y Meave, 2008; Derroire et al., 2016). La capacidad de recuperación de este ecosistema posterior al disturbio es fundamental para la conservación de su biodiversidad. Ewel (1977), basado en las diferencias ecológicas entre los bosques secos y húmedos, propuso un modelo de resiliencia para el BTC definido por una composición florística más simple, con pocas etapas sucesionales, de mayor rapidez en cuanto a la recuperación de sus atributos estructurales hacia la condición de bosque maduro; debido a su baja estatura exhibida y a la alta capacidad de producir rebrotes (Murphy y Lugo, 1986).

El patrón de cambios en la composición y estructura de las sucesiones es el punto de partida para conocer el potencial de resiliencia de un bosque (Derroire et al., 2016). En este sentido, estudios de crono secuencias, en los cuales se usan sitios con diferentes edades de abandono para inferir el proceso sucesional, han mostrado ser útiles para describir los patrones básicos en la regeneración del bosque en campos agrícolas típicos del trópico americano (Quesada et al., 2009). Resultados de estudios por lo general han mostrado un incremento progresivo en cuanto a la riqueza de especies (Sheil y Bursalem, 2003; Derroire et al., 2016), altura (Kalacska et al., 2004; Leirana-Alcocer, HernándezBetancourt, Salinas-Peba y Guerrero-González, 2009), área basal y biomasa aérea (Becknell, Kucek y Powers, 2012), así como una marcada diferencia entre la composición florística y el estadio sucesional más reciente al más antiguo (Lebrija-Trejos et al., 2008). Otros por el contrario han evidenciado el comportamiento errático de las abundancias de árboles (Chazdon et al., 2007) y una riqueza de especies y biomasa aérea que se recuperan lentamente en comparación con el bosque original (Derroire et al., 2016). En consecuencia, el BTC ha mostrado poseer menor capacidad de resiliencia a los impactos antropogénicos comparado con el bosque húmedo, en donde se han observado tasas de recuperación que se encuentran entre 30 años y 60 años (Poorter et al., 2016).

En Chiapas, la distribución del BTC actualmente ocurre en las regiones: Planicie Costera (Miranda, 1952), Sierra Madre (López-Toledo et al., 2012) y la Depresión Central (Reyes-García y Sousa, 1997). Esta última, considerada la región más críticamente amenazada del Pacífico mexicano, ya que más de $95 \%$ de la superficie de 
distribución original del BTC ha sido sustituida por terrenos de uso agropecuario (Ceballos et al., 2010). En esta región la información básica que permita describir el proceso de sucesión del BTC es muy limitada. Los escasos estudios realizados particularmente en los bosques secundarios registran un riqueza de especies entre 119 y 157 especies árboreas en fragmentos con menor nivel de pertubación (Espinosa-Jiménez et al., 2014; Sánchez-Molina, 2014). Mientras, la composición florística de los remanentes menos perturbados esta conformada por especies árboreas tardías, cuyos ensamblajes cambian por el efecto combinado de la heterogeidad ambiental y el disturbio antrópogenico (Rocha et al., 2014). Prácticamente, no se dispone de estudios que documenten los cambios y procesos ecológicos de la sucesión frente al disturbio antrópico, los cuales sirvan de base para comprender el fenómeno y planificar las acciones de conservación y restauración del BTC en esta región.

\section{OBJETIVOS}

$\mathrm{El}$ próposito de este estudio fue evaluar los cambios en la estructura, diversidad y la composicion florística de la comunidad árborea en el BTC de la Depresión Central de Chiapas, en sitios de potreros con diferentes edades de abandono y tipos de disturbio humano. La hipótesis por confirmar fue que la estructura del bosque secundario es resiliente, con una recuperación gradual en cuanto a la riqueza de especies, diversidad, altural, área basal y biomasa áerea conforme avanza el tiempo de abandono, pero con una menor semejanza en su composición florística.

\section{MATERIALES Y MÉTODOS}

\section{Área de estudio}

Este trabajo se realizó en el sector suroeste de la reserva forestal Vedada de Villa Allende, en los municipios de San Fernando, Berriozábal y Tuxtla Gutiérrez, Chiapas (Sur de México), entre los paralelos $16^{\circ} 47^{\prime}$ N”- 93²' $12^{\prime}$ y $16^{\circ} 48^{\prime} \mathrm{S}$ 911' E (Fig. 1). El tipo de clima dominante (clasificación de Köppen modificada por García, 2004) es el cálido subhúmedo, con lluvias en verano, temperatura media anual de $22{ }^{\circ} \mathrm{C}$ y precipitación total anual de $955.8 \mathrm{~mm}$ (López, 2006). Los estratos geológicos están representados por rocas sedimentarias del Cretácico Superior y Eoceno integradas por caliza, lutita y arenisca (Instituto Nacional de Estadística y Geografía [Inegi], 1985). El suelo es de tipo litosol de textura media y feozem; con uso agrícola dominante, pero con una fuerte tendencia a transitar al uso urbano (Secretaria de Medio Ambiente y Vivienda [Semavi], 2009). Sobre lomeríos de suaves pendientes se desarrolla de forma predominante el BTC (sensu Rzedowski, 1991) y en menor extensión el bosque tropical subcaducifolio y bosque de Quercus.

\section{Selección de los sitios de muestreo}

Se seleccionaron campos abandonados que previamente habían tenido un uso ganadero, con características similares en sustrato geológico y posición topográfica. Los tiempos de abandono se estimaron en una primera instancia con los datos de encuestas y recorridos con habitantes locales y después fueron corroboradas mediante fotointerpretación de imágenes satelitales Landsat TM (1992 y 2000), Spot 5 (2009), ortofotos de 2001 (a blanco y negro, escala 1:75 000) y 2007 (a color, escala 1:40 000); fotografías aéreas de 1972 (a blanco y negro, escala 1:50 000) y datos satelitales Landsat 2015 consultados en la plataforma de Google Earth. Se definieron cuatro condiciones de tiempos de abandono posterior a la perturbación: a) 10 años (C10), sitio usado para pastoreo hasta el año de 1992 (existen algunos árboles del bosque original), desmontado nuevamente en 2009 y excluido por cercado desde 2011 para establecer la reserva ejidal, perturbado por prácticas de limpia con eliminación de la regeneración natural; b) 19 años (C19), se cultivaron pastizales por más de 46 años, su ultimó ciclo de uso fue 2000, perturbada por la constante recolección de leña; c) 35 años (C35), se usó como agostadero hasta que se abandonó en 1982, perturbada ocasionalmente por incendios forestales (último en 2007) y extracción de postes; d) Mayor a 40 años (C40), el lugar fue utilizado por 30 años para el ganado sin eliminar totalmente el bosque, la actividad se abandonó a finales de los setenta, sitio poco perturbado, eventualmente se recolecta leña. 

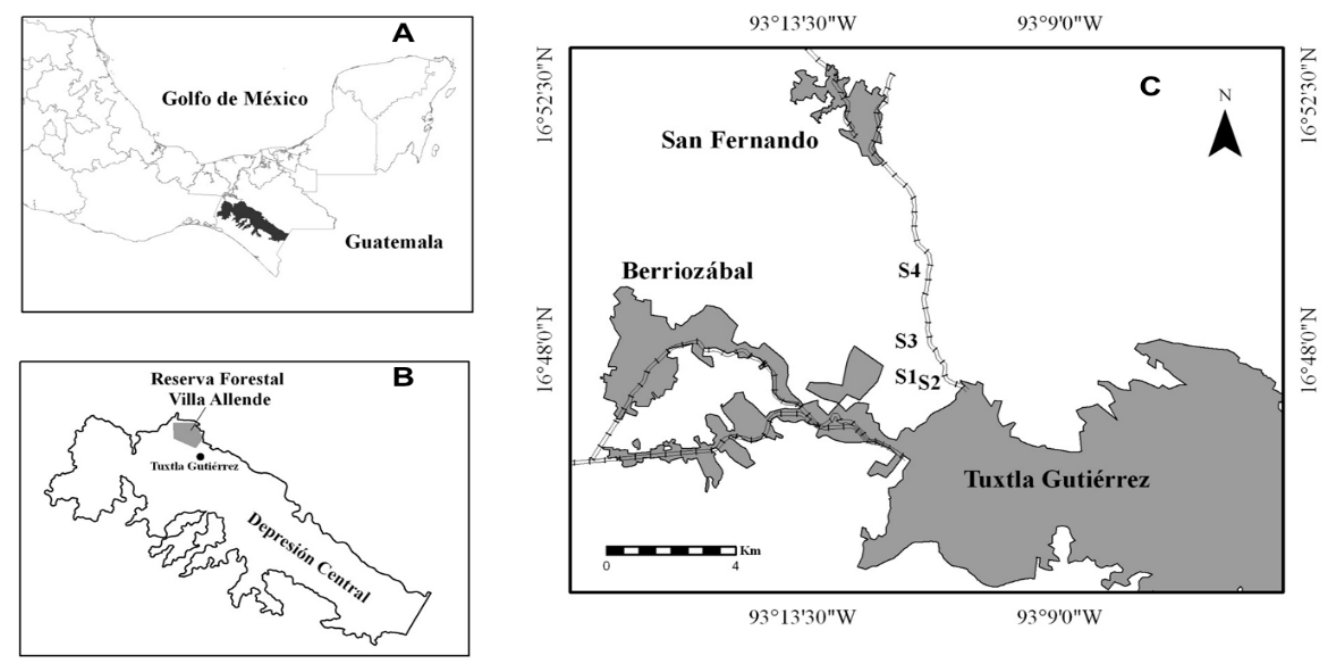

FIgura 1. Localización de la Depresión Central, Chiapas, México (A), Reserva Forestal de Villa Allende (B) y los sitios de muestreo (C).

Condiciones de abandono: S1 = 35 años, S2 = 40 años, S3= 19 años, S4 = 10 años.

\section{Muestreo de la vegetación}

Se midieron 20 unidades de muestreo (UM) de $1000 \mathrm{~m}^{2}$ (20 $\mathrm{m} \times 50 \mathrm{~m})$. Para la asignación del tamaño de muestra a cada condición de abandono, se empleó el protocolo propuesto por Kalacska et al. (2004), en el que se recomienda medir un mínimo de tres parcelas por cada edad de abandono; debido a que algunas condiciones de abandono fueron más frecuentes que otras, se les asignaron UM adicionales, quedando la muestra distribuida de la siguiente manera: cinco UM en C10, seis en C19, seis en C35 y tres en C40. Se estimó además la completitud del inventario florístico mediante la comparación de la riqueza observada y estimada a través de indicadores no paramétricos (Colwell, 2009). En cada UM se midió el diámetro a la altura del pecho (DAP) y la altura total (del suelo al ápice del árbol), de todas las plantas leñosas con DAP mayor a $5 \mathrm{~cm}$ y altura mayor a $1.30 \mathrm{~m}$. También se identificó la especie botánica de cada una de las plantas medidas. Se colectaron muestras botánicas de los individuos que no fueron identificados en campo, las cuales se determinaron mediante comparación usando ejemplares de la colección de plantas vasculares del Herbario Eizi Matuda (HEM) de la Universidad de Ciencias y Artes de Chiapas [Unicach], Herbario de la Secretaría de
Medio Ambiente e Historia Natural (CHIP) y por consulta a especialistas de algunas familias. Se siguió el sistema de clasificación APGIV (Angiosperm Phylogeny Group, 2016) angiospermas. Los nombres taxonómicos fueron corroborados con la base de datos The Plant List (2013).

\section{Análisis de datos}

Se generaron curvas de especie-área a partir de las cuales se obtuvo la riqueza rarificada $\left(\mathrm{S}_{o b s}\right)$ por condición de abandono en una superficie determinada. Se estimó también la riqueza esperada $\left(S_{\text {est }}\right)$ mediante el uso indicadores no paramétricos basada en 100 aleatorizaciones sin reemplazo, eligiendo para cada condición de abandono los que se ajustaran con mayor precisión a la $\mathrm{S}_{\text {est }}$ Todos los cálculos se realizaron en el programa EstimateS v. 8.2.0 (Colwell, 2009). Se calculó la diversidad de especies de cada condición de abandono mediante los siguientes índices de diversidad $\alpha$ :

recíproco de Simpson $\left(D_{2}=\frac{1}{\sum_{i=1}^{S} p_{i}^{2}}\right)$,

Shannon-Wiener $\left(H=\sum_{i=1}^{S} p_{i} \log _{b} p_{i}\right)$ 
y su respectiva medida de equitatividad $\left(\mathrm{J}=\frac{H}{\log (S)}\right)$

y diversidad verdadera $\left({ }^{1} \mathrm{D}=\exp (\mathrm{H})\right)$;

donde:

$p_{i}=$ abundancia relativa de la especie $i$

$\mathrm{S}=$ número de especies que componen la comunidad

$\log _{b}=\operatorname{logaritmo}$ en base b de $p_{i}$ (Brower, Zar y Ende, 1998;

Magurran, 2004) y

${ }^{1} \mathrm{D}=$ diversidad verdadera (Jost, 2006)

Este último índice fue calculado mediante el programa SpadeR (Chao et al., 2016).

Para caracterizar la dominancia de las especies por condición, se calculó el índice de valor de importancia relativo de cada una de las especies midiendo y sumando los valores de densidad (número de individuos por especie/total de individuos de las especies $\times 100)$, frecuencia (número de unidades de muestra en el que contabilizó una especie/el número total de unidades de muestra $\times 100$ ) y dominancia (área basal de cada especie/total del área basal de todas las especies $\times 100$ ) registrados en cada clase de vegetación analizada (MuellerDombois y Ellenberg, 2002).

Se calcularon los valores promedio de las siguientes métricas de los atributos estructurales entre las diferentes condiciones de tiempo de abandono: densidad de individuos (ind $\mathrm{m}^{2}$ ), altura máxima (de los 10 individuos más altos; $\mathrm{m})$, área basal $\left(\mathrm{m}^{2} \mathrm{ha}^{-1}\right)$ y biomasa área $\left(\mathrm{Mg} \mathrm{ha}^{-1}\right)$. La biomasa aérea se cuantificó mediante la aplicación del modelo alométrico propuesto por Martínez-Yrizar et al. (1992) para el bosque seco de México:

$$
\begin{aligned}
\log _{10}(\text { mass })= & a+0.9011\left(\log _{10} A B\right) \\
& +0.5715\left(\log _{10} D M\right) \\
& +0.5654\left(\log _{10} H\right)
\end{aligned}
$$

donde:

mass $=$ biomasa aérea $(\mathrm{kg})$

$a$ es la constante $=-0.7590$

$A B$ : área basal $\left(\mathrm{cm}^{2}\right)$

$D M$ : densidad de la madera $\left(\mathrm{g} / \mathrm{cm}^{3}\right)$

$H$ : altura (m)
La altura se tomó directamente de los datos del inventario de campo. La densidad de madera para cada especie se obtuvo de la consulta a la Base de Datos de Atributos Funcionales y Ecológicos de los Árboles (World Agroforestry [Icraf], s/f). Se consideraron los valores promedio de la densidad de madera a escala de familia o género para las especies que no contaron con registro.

Los atributos de la estructura se analizaron con una prueba de Shapiro-Wilks para determinar la premisa de normalidad; todas las variables se ajustaron a este comportamiento. Por lo tanto, se aplicó un análisis de varianza de una sola vía (ANOVA, en donde cada condición representó un tratamiento y cada parcela una repetición), para identificar las diferencias entre los atributos y los tiempos de abandono. Cuando los valores del ANOVA fueron significativos $(p \leq 0.05)$, se realizaron pruebas de comparación múltiple de Tukey a posteriori, para conocer cuáles de los tratamientos eran estadísticamente diferentes. Este mismo procedimiento se realizó para determinar si el tiempo de abandono afecta la riqueza acumulada y en el índice de diversidad verdadera $\left({ }^{1} \mathrm{D}\right)$ en cada condición de estudio.

Las diferencias significativas entre los índices de recíproco de Simpson (1/D), Shannon-Wiener $(\mathrm{H})$ y equitatividad (J) con las condiciones de abandono, se obtuvo a través de una prueba de Kruskal-Wallis. Con la finalidad de conocer las diferencias estadísticas entre los tratamientos, se obtuvo la comparación de suma de rangos (U de Mann-Witney, $p<0.05$ ), bajo la hipótesis nula de igualdad de condiciones (Gardner et al., 2012).

La variación en la composición arbórea entre las diferentes condiciones de abandono se analizó a través de los valores promedios del índice de Beta de Bray-Curtis $\left(\mathrm{S}_{\mathrm{B}}\right),\left(\mathrm{S}_{\mathrm{B}}\right)=\Xi\left(X_{i j}-X_{i k}\right) / \Xi\left(X_{i j}+X_{i k}\right)$, donde $X i j$ es la abundancia de la especie $i$ en el sitio $j$ y $X i k$ es la abundancia de la especie $i$ en el sitio $k$. Los valores de esta medida oscilan de 0 a 1; el valor 0 indica total identidad y el valor 1 unidades sin especies en común. Previo a este análisis, la matriz de parcelas de muestreo y sus correspondientes especies se estandarizó mediante su transformación a una escala de raíz cuadrada. Posteriormente, se realizó un 
análisis de ordenamiento no métrico multidimensional (NMDS) (Minchin, 1987), el cual integró las parcelas de muestreo con base en la composición de especies. En la ordenación se utilizó una matriz de semejanza entre las parcelas de muestro pertenecientes a las cuatro condiciones de abandono, la cual contenía los valores del índice de BrayCurtis. Para determinar las diferencias estadísticas en las dimensiones de ordenación de las especies entre las condiciones de abandono se empleó una prueba de análisis de varianza multivariado permutacional (ADONIS, por sus siglas en inglés; McArdle y Anderson, 2001) usando la matriz de distancias, bajo la hipótesis nula de igualdad entre la composición arbórea. Se aplicaron pruebas a posteriori tipo Bonferroni para identificar a los grupos de especies que difirieron entre sí sobre estas dimensiones de la ordenación. Finalmente, todos los análisis estadísticos se realizaron en $\mathrm{R}$ versión 3.5.3 (R Core Team, 2019).

\section{Resultados}

\section{Composición florística}

Se registró un total de 142 especies correspondientes a 96 géneros y 41 familias. Las familias con un mayor número de especies fueron Leguminoseae (35), Euphorbiaceae (10), Rubiaceae (10) y Compositae (9). El resto de las familias contribuyó con seis o menos especies. Lonchocarpus fue el género mejor representado con $4.2 \%$ del total, seguido de Eugenia con 3.5\%, Acacia, Bursera, Cordia y Leucaena, todos estos con $2.8 \%$. Diecisiete especies concentraron la mitad de las abundancias totales, de las cuales Montanoa tomentosa Cerv. con 129 individuos $(5.1 \%$ del total de individuos muestreados), Tecoma stans (L.) Juss. ex Kunth. con 123 (5\%), Perymenium grande Hemsl. con 117 (4.7\%), Eugenia savannarum Standl. \& Steyerm. con 109 (4\%) y Machaerium arboreum (Jacq.) Benth. con 102 (4\%) fueron las más abundantes.

Las comparaciones de la similitud florística entre las condiciones de abandono según el índice de Bray-Curtis mostraron una casi total disimilitud entre los bosques de edad más avanzada ( $88 \%$ a $92 \%$ ) frente al bosque de 10 años de abandono, así como entre las condiciones de abandono C35 (79\%) y C40 (83\%) con respecto al bosque de 19 años. La mayor semejanza florística (66\%) se presentó entre los bosques de C35-C40 (Fig. 2). En correspondencia a estos resultados, el análisis de escalamiento multidimensional no-métrico (NMDS) reveló la conformación de tres grupos ecológicos estadísticamente diferentes (estrés: 0.13; Fig. 3). El primer grupo (A) estuvo integrado por las parcelas del bosque con 10 años de abandono, el segundo (B) por cinco parcelas del bosque con 19 años de abandono y el tercer (C) grupo estuvo conformado por una parcela de 19 años de abandono con todas las parcelas correspondientes a los bosques de 35 años y $>40$ años de abandono. El ADONIS mostró diferencias significativas ( $\not$ $<0.05)$ en la composición arbórea entre todos los grupos ecológicos formados previamente en el NMDS (Tabla 1). Esta tendencia muestra la complementariedad existente entre la composición de especies de los sitios de más edad de abandono (35 años y > 40 años de abandono) contra las condiciones de más reciente abandono (C19 y C10).

\section{Riqueza, diversidad y estructura}

La riqueza acumulada mostró diferencias significativas con el tiempo de abandono $\left(\mathrm{F}_{3}=7.1, p<0.05\right)$, especialmente entre las condiciones C10-C35 (Fig. 4a). En esta última condición de abandono, también se registró el mayor número de especies acumuladas (98). Esta misma tendencia siguió la riqueza esperada, cuyo valor más alto se concentró en las parcelas con 35 años de abandono (Fig. 4d). De igual manera, se encontraron diferencias significativas en la diversidad únicamente entre los índices $\mathrm{H}^{\prime}$ (Kruskall-Wallis $\left.x^{2}=12.54, p<0.05\right)$ y ${ }^{1} \mathrm{D}\left(\mathrm{F}_{3}=7.3, p<0.05\right)$ con las condiciones C10 y C35 (Tabla 2). El resto de los índices de dominancia y equitatividad no mostraron diferencias estadísticas significativas. 


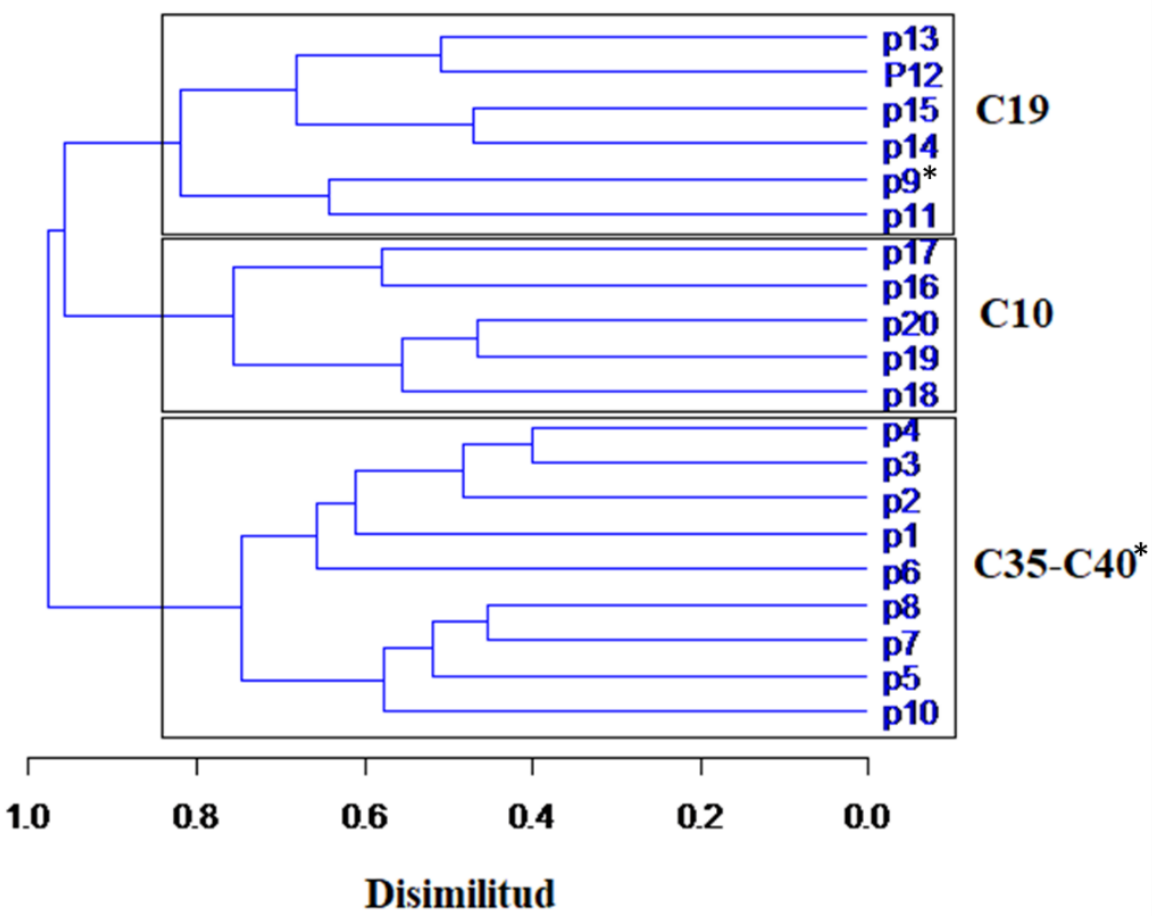

FIGURA 2. Dendograma de agrupamiento jerárquico con base en el índice de disimilitud de BrayCurtis.

Se agruparon el número de parcelas (P) correspondiente a cada condición de abandono (líneas negras incertadas en el dendrograma) en el área de estudio.

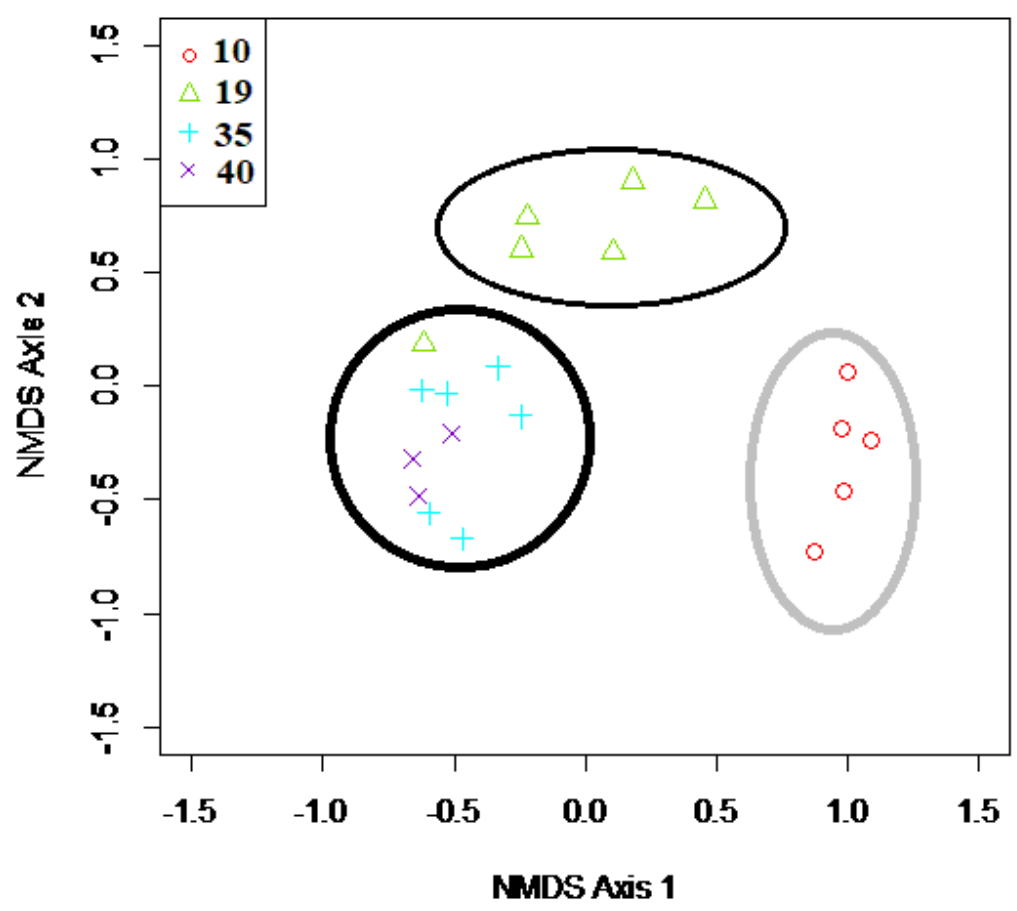

FIGURA 3. Ordenación multidimensional no-métrica basado en la raíz cuadrada de las abundancias de todas las especies y por condición de abandono (años).

Las líneas indican la afinidad de parcelas de acuerdo con las abundancias: Grupo A: línea negra delgada, Grupo B: línea negra gruesa, y Grupo C: línea gris. 
TABLA 1. Pruebas de diferencias en la composición arbórea entre los grupos ecológicos con base en el procedimiento de ADONIS, en la Depresión Central, Chiapas, México.

\begin{tabular}{cccc}
\hline $\begin{array}{c}\text { Comparación entre grupos } \\
\text { ecológicos }\end{array}$ & $F$ & $R^{2}$ & Valor de $p$ \\
\hline Grupo A vs Grupo B & 6.58 & 0.46 & 0.008 \\
Grupo A vs Grupo C & 3.8 & 0.22 & 0.001 \\
Grupo B vs Grupo C & 6.59 & 0.33 & 0.001 \\
\hline
\end{tabular}

Significancia estadística: $p<0.05$; corrección de Bonferroni valor de $\alpha$ ajustado: 0.016.
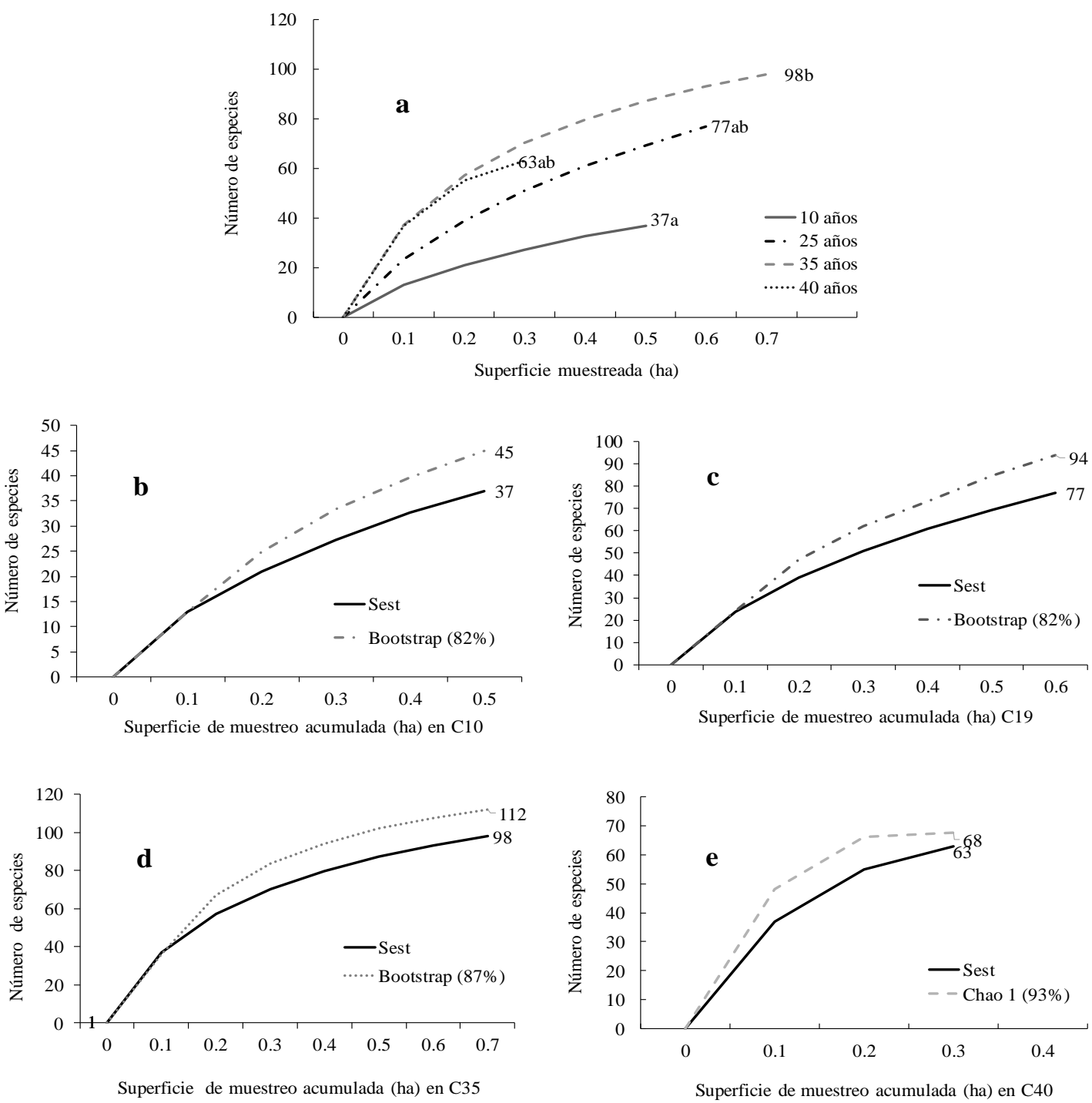

Figura 4. Curvas de especie-área por tiempo de abandono en la Depresión Central, Chiapas, México. a) Riqueza acumulada.

Letras diferentes entre curvas indican diferencias significativas (Tukey, $p<0.005$ ); b, c, d y e riqueza esperada mediante los estimados noparamétricos con mayor precisión. 
TABLA 2. Valores promedio y desviación estándar (entre paréntesis) de los atributos estructurales y diversidad por tiempo de abandono de los bosques secundarios en la Depresión Central, Chiapas, México.

\begin{tabular}{|c|c|c|c|c|c|c|}
\hline Condiciones & $H^{\prime *}$ & ${ }^{1} D$ & $\begin{array}{l}\text { Densidad } \\
\text { (ind. } h a^{-1} \text { ) }\end{array}$ & $\begin{array}{l}\text { Altura máxima } \\
\qquad(\mathrm{m})\end{array}$ & $\begin{array}{c}\text { Área basal } \\
\left(m^{2} h a^{-1}\right)\end{array}$ & $\begin{array}{c}\text { Biomasa área } \\
\left(\mathrm{Mg} \mathrm{ha}^{-1}\right)\end{array}$ \\
\hline 10 años & $1.7(0.8)^{a}$ & $8.5(0.6)^{a}$ & $410(99)^{a}$ & $6.7(0.5)^{a}$ & $6.5(2.8)^{a}$ & $16.0(7.1)^{\mathrm{a}}$ \\
\hline 19 años & $2.6(0.2)^{\mathrm{abc}}$ & $13.8(4.1)^{\mathrm{ab}}$ & $1341(398)^{\mathrm{b}}$ & $10.2(1.23)^{\mathrm{bc}}$ & $11.1(4.7)^{\mathrm{ab}}$ & $25.8(9.8)^{\mathrm{ab}}$ \\
\hline 35 años & $3.0(0.3)^{b c}$ & $21.7(6.8)^{\mathrm{b}}$ & $1581(423)^{b}$ & $9.08(2.19)^{\mathrm{ab}}$ & $16.7(3.5)^{\mathrm{b}}$ & $37.7(11.0)^{\mathrm{b}}$ \\
\hline > 40 años & $2.8(0.2)^{\mathrm{abc}}$ & $18.1(4.9)^{a b}$ & $1730(384)^{\mathrm{b}}$ & $12.2(0.0)^{c}$ & $15.0(0.6)^{b}$ & $42.8(0.7)^{\mathrm{b}}$ \\
\hline
\end{tabular}

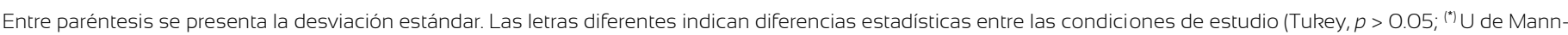
Whitney, $p<0.05$ ).

La abundancia total de árboles en las cuatro condiciones de estudio fue de 2488 individuos. La condición C40 registró la mayor cantidad de árboles por hectárea y solo se encontraron diferencias significativas entre C10 y el resto las condiciones de abandono (Tabla 2). En los casos de altura y biomasa aérea, los valores más altos se registraron en la condición C40, mientras que en el área basal el valor mayor se presentó en C35. En todas se encontraron diferencias significativas $(p>0.05)$ contra el tiempo de abandono, pero estas fueron más notables entre las condiciones C10 y C40 (Tabla 2).

El ensamblaje de especies más importantes en la estructura fue consistente entre las condiciones C25, C35 y C40, aunque mostró gran variación en la condición de 10 años. De acuerdo con los valores del IVIR acumulado, Luehea candida (Moç. \& Sessé ex DC.) Mart. dominó la condición C10 con 33.52\%. Mientras que T. stans, Lysiloma acapulcense (Kunth) Benth., M. tomentosa y E. savannarum fueron las especies con los valores más altos de IVIR en el resto de las condiciones de estudio (Suplemento). En cuanto a la exclusividad de especies, el mayor número de estas se registró en la condición de 19 años (27 especies), seguida de la condición de 35 años (21 especies); y la menor cantidad de especies exclusivas se registró en C40 (Suplemento).

\section{DISCUSIÓN}

La hipótesis nula, que plantea el incremento de la riqueza, diversidad y estructura del bosque tropical caducifolio con el tiempo de abandono, se rechaza pues en todos los atributos evaluados solo se encontraron diferencias estadísticas altamente significativas entre las condiciones de edad más avanzada (35 años y $>40$ años de abandono) con la categoría de 10 años (Tabla 2). Por el contrario, cada atributo analizado siguió sendas distintas, lo cual coincide con los resultados encontrados en estudios recientes para el bosque tropical, en los cuales se descarta a la edad entre los principales factores determinantes de los cambios durante el proceso de sucesión (Dupuy et al., 2012; Norden et al., 2015). Así también, se evidencia una lenta recuperación de la composición florística (Poorter et al., 2016; Derroire et al., 2016; Beltrán-Rodríguez et al., 2018). A continuación, se discuten los resultados encontrados en cada atributo:

\section{Composición florística}

En cuanto a número de especies, Leguminosae y todos los géneros mejor representados en el área de estudio son resultados que concuerdan con el patrón de elementos florísticos dominantes para BTC de México (Rzedowski y Calderon, 2013, Vargas et al., 2015). En términos de las abundancias, la composición florística de los bosques está preponderadamente representada por especies muy tolerantes al disturbio y la sequía. Es sabido que T. stans es una especie pionera que progresa fácilmente en áreas alteradas con suelos pedregosos y altamente resistente al estrés hídrico (Vázquez-Yanes et al., 1999). También, M. tomentosa es una especie típica del bosque secundario que posee una alta capacidad de colonizar sitios de condición 
muy perturbada como son los suelos con altas concentraciones de nitrógeno (Gelviz-Gelvez y Pavón, 2013).

La disimilitud arbórea entre las condiciones de estudio, en general, fue alta $(79 \%$ a $92 \%)$ entre las condiciones de menor tiempo de abandono (C10 y C19) comparada con las de mayor tiempo de abandono (C35 y C45). Estos resultados concuerdan con los hallados en diversos BTC secundarios de México, en los cuales se apoya la existencia de una alta tasa de recambio de especies entre los sitios (Lebrija-Trejo et al., 2008, Almazán-Núñez, Arizmendi, Eguiarte y Corcuera, 2012, Beltrán-Rodríguez et al., 2018). Con base en el valor de $p$ de la prueba significativa basada en el ADONIS, se confirma que la composición de especies cambia con el tiempo de abandono y se demuestra que las condiciones de menor edad de abandono (C10 y C19) son florísticamente diferentes entre sí y con respecto a las de mayor edad de abandono (C35-C40). Resultados similares han sido registrados en otros bosques secundarios del Neotrópico, sugiriendo que la recuperación de la composición de las especies en estos bosques cambia lentamente con el tiempo (Mora et al., 2015; Lebrija-Trejo et al., 2010; Derroire et al., 2016). Aunque las tasas de recuperación de la composición de especies de los nuevos ensamblajes son atribuidas a la disponibilidad de semillas de la vegetación remanente (Norden et al., 2015), el disturbio también es un factor importante que ha propiciado variaciones en las condiciones ambientales que influyen en las tasas de recuperación de las especies del área de estudio (Rocha-Loredo et al., 2010). Asimismo, la semejanza de especies entre las condiciones de mayor tiempo de abandono puede explicarse debido a la cercanía entre los sitios muestreados (López-Jiménez, Duran-García y Dupuy-Rda, 2019).

\section{Riqueza, diversidad y estructura}

La riqueza acumulada y diversidad alcanzaron valores altos en la etapa intermedia a 35 años, lo cual se aproximan a lo observado para bosques secundarios en otras regiones del país (Oaxaca, 141 especies, H' 3.34, diámetro mínimo $\geq 1.0$,
Lebrija-Trejo et al., 2008; Morelos, 79 especies, H' entre 2.39 y 2.73 , diámetro mínimo $\geq 2.5 \mathrm{~cm}$, Beltrán-Rodríguez et al., 2018) e incluso al bosque conservado ( $\mathrm{H}^{\prime}$ entre $2.9 \mathrm{y}$ 4.17 , diámetro mínimo $\geq 2.5 \mathrm{~cm}$, Trejo y Dirzo, 2002). Como ya se ha demostrado para otros bosques secundarios neotropicales, los estados intermedios parecen superar en riqueza y diversidad a los estados avanzados o inclusive a los bosques conservados a causa del proceso de establecimiento y persistencia de especies pioneras favorecidas por el disturbio (Sheil y Brusalem, 2003). Este pico máximo de acumulación de especies consistente con la hipótesis del disturbio intermedio descrita por Connell (1978), se descarta para el área de estudio, debido a que no se registraron diferencias estadísticas entre C35 y C40. Por lo tanto, la mayor acumulación de especies en C35 se explica por la confluencia de especies de aparición temprana con las de etapas avanzadas (Lebrija-Trejos et al., 2010), la alta semejanza florística entre las condiciones de mayor edad y debido a las perturbaciones, las cuales pudieron crear condiciones ambientales favorables para la presencia de especies de alta tolerancia al disturbio (Kalacska et al., 2004) y limitar la regeneración de especies propias del bosque maduro vía dispersión y banco de semillas en la condición de abandono más avanzada (Hammond, 1995).

La densidad de plantas presentó un comportamiento diferente a lo mostrado en otros BTCs neotropicales, pues no llegó a estabilizarse en las primeras condiciones de abandono (Mora et al., 2015; Lebrija-Trejos et al.; 2010; Kalacska et al., 2004). De acuerdo con Chazdon et al. (2007), los cambios en la densidad de plantas del bosque seco son generalmente inconsistentes al tiempo de abandono, debido a que estos están influenciados por factores como la composición de especies, denso-dependencia y el disturbio. El incremento gradual de la densidad arbórea en el área de estudio se debió principalmente a la contribución del alto número de jóvenes individuos arbóreos $(5 \mathrm{~cm}-10$ $\mathrm{cm}, 80 \%$ ) coexistiendo en los bosques de edad intermedia $(\approx 35$ años y 40 años). Individuos, por lo general, pertenecientes a especies con alta capacidad de crecimiento 
y adaptación a las actuales condiciones de disturbio (Almazán-Núñez et al., 2012; Lebrija-Trejos et al., 2010).

El intervalo de altura máxima de los árboles (9 m - 12 m) registrado para el área de estudio mostró un aumento conforme al tiempo de abandono (Tabla 2), con valores aproximados a los observados para los bosques secundarios en Yucatán (Dupuy et al., 2012, $\approx 10 \mathrm{~m}-12 \mathrm{~m}$ ) y al BTC conservado en México ( $\approx 8 \mathrm{~m}-12 \mathrm{~m}$, Trejo, 1998). En bosques secundarios neotropicales se han observado que la recuperación de su altura depende de la historia de vida de las especies dominantes durante la sucesión, en las etapas tempranas las especies pioneras emplean una estrategia de colocación temprana para la adquisición de recursos, crecer rápido y dominar pronto el dosel (Guariguata y Ostertag, 2001). Los datos del presente estudio coinciden con este planteamiento, porque especies pioneras como $M$. tomentosa, Cocblospermum vitifolium (Willd.) Spreng., Heliocarpus terebinthinaceus (DC.) Hochr. y Baubinia divaricata L., dominaron dosel en C10, C19 y C35.

El área basal y la biomasa aérea mostraron una tendencia monótona a aumentar con el tiempo de abandono. De manera general se sabe que estos parámetros estructurales están correlacionados y suelen estabilizarse en las etapas tempranas de sucesión, para luego continuar acumulándose de forma lenta por varias décadas más antes de alcanzar valores aproximados a los bosques conservados (Chazdon et al., 2007). Diversos estudios en BTCs de México, han mostrado que ambos atributos tienden a acumularse rápidamente en las etapas tempranas de la sucesión [Lebrija-Trejos et al. (2008): $\geq 1 \mathrm{~cm}$ de DAP, 15 $\mathrm{m}^{2} /$ ha; Dupuy et al. (2012): $>5 \mathrm{~cm}$ de DAP, $20 \mathrm{~m}^{2} /$ ha, Read y Lawrence (2003): $\geq 1 \mathrm{~cm}$ de DAP, $34 \mathrm{~m}^{2} /$ ha; Rozendaal et al. (2016): $31.0 \mathrm{mg} \mathrm{ha}^{-1} \pm 25.1 \mathrm{mg} \mathrm{ha}^{-1}$, hasta alcanzar el promedio máximo después de 3 a 5 décadas. Los resultados de este estudio tuvieron un comportamiento contrario a estos planteamientos, debido a que ambos parámetros registraron valores bajos comparados con los bosques previamente citados. Esta situación podría deberse al efecto acumulado del uso agrícola del suelo en la región sobre la composición de especies (Marin-Spiotta, Cusak, Ostertag y Silver, 2007) y a las distintas perturbaciones actuales (extracción de madera, incendios, etc.), las cuales han propiciado una recuperación lenta del área basal y la biomasa área.

El índice estructural IVIR mostró que $L$. candida fue la especie más importante en la condición de abandono más joven, lo cual se explica en la alta capacidad de adaptación que posee esta especie a los ambientes perturbados (Cordero, 2003) y por la contribución de árboles remanentes, producto del fomento por parte de los productores para favorecer vegetación que proteja la vertiente de agua en el ejido Viva Cárdenas. Los bosques de 19 años y 35 años de abandono estuvieron dominados por las especies heliófilas T. stans y M. tomentosa. En cambio, la condición de edad más avanzada estuvo dominada por especies tardías pertenecientes a los géneros Eugenia y Machaerium, registrados por Rocha et al. (2010) para la Depresión Central.

\section{Resiliencia de los bosques secundarios}

En el presente estudio se encontró una rápida recuperación de la riqueza arbórea frente una lenta recuperación de la estructura y la composición florística. La resiliencia del BTCs, representada por la alta capacidad de recuperación en términos de diversidad y riqueza de especies en las primeras etapas de la sucesión, se debe a la gran cantidad de especies con dispersión anemócora (Hilje, Calvo-Alvarado, Jiménez-Rodríguez, Sánchez-Azofeifa, 2015), que presentan mayor capacidad de crecer y progresar en ambientes perturbados (Vieira y Scariot, 2006; Bongers, Pooter, Hawthorne y Sheil, 2009). En cambio, la menor capacidad de recuperación de la estructura y composición de los bosques en la reserva, coinciden con los planteamientos de Mora et al. (2014), Derroire et al. (2016) y Poorter et al. (2016), al señalar que estos bosques poseen una menor resiliencia en comparación con los bosques húmedos, debido a que resultan ser más sensibles a los factores de sitio, tales como el uso previo del suelo, tipo e intensidad de la perturbación. La Depresión Central posee un largo historial de disturbio, hasta antes del siglo XX estuvo sometida a una intensa explotación forestal (Vargas, 
Escobar y Del Ángel, 2000) y asociada con el uso rotativo de cultivos agrícolas-ganadería extensiva. A su vez, la escasa presencia de individuos arbóreos de tallas grandes $(<60$ $\mathrm{cm})$ producto de la tala clandestina, aún observada durante los recorridos de campo, es un indicador proxy (Rüger, Williams-Linera, Kissling y Huth, 2008) de los cambios negativos de las perturbaciones sobre la estructura y resiliencia de estos bosques.

\section{CONCLUSIONES}

Los bosques caducifolios secundarios de la Depresión Central fueron diferentes en diversidad, estructura y composición florística, entre las condiciones tempranas con las de mayor tiempo de abandono. En consecuencia, la mayoría de los atributos ecológicos evaluados no siguieron el mismo patrón conforme el tiempo de abandono postdisturbio, de manera que las tasas de recuperación de los bosques no siguieron una trayectoria unidireccional. Por otro lado, los bosques secundarios con mayor tiempo de abandono (C35-C40) presentaron la riqueza específica más alta confirmando (resiliencia alta) la importancia ecológica que tienen para resguardar la diversidad arbórea local. Por el contrario, la composición y estructura mostraron una recuperación lenta (resiliencia baja), debido a las distintas perturbaciones presentes en las condiciones de abandono, el tiempo de abandono postdisturbio, así como por la dominancia de un grupo de especies generalistas tolerantes al disturbio.

Por último, se recomienda que los planes acción de la reserva en futuro incorporen los bosques en sus diferentes etapas de sucesión. Los BTCs son importantes fuentes de propágulos, base para los programas de conservación y restauración, los cuales además requieren de estudios más detallados para conocer el efecto de las perturbaciones sobre la dinámica sucesional y la resiliencia de los bosques, los cuales se encuentran en constante amenaza por las actividades humanas.

\section{RECONOCIMIENTOS}

Se agradece a las autoridades locales y campesinos por los permisos para realizar el trabajo de campo en la reserva forestal Villa Allende. A la Secretaria de Medio Ambiente e Historia Natural por el financiamiento parcial otorgado al primer autor a través del proyecto "Investigación de la Biodiversidad en Chiapas (clave: 042151S09201E10A003000), así como por el apoyo brindado en la gestión de permisos de colecta, logística y acceso al herbario (Herbario CHIP). A los curadores $\mathrm{M}$ en C. Oscar Farrera Sarmiento (Herbario CHIP) y Biól. Josefa Anahí Espinosa Jiménez (Herbario Eizi-Matuda, Unicach) por su apoyo en la identificación taxonómica de las colectas botánicas. A los estudiantes Daniel Pérez Muñoz y Dalia Elizabeth Sántiz Gómez por su apoyo en la adquisición de los datos de campo.

\section{REFERENCIAS}

Almazán-Núñez, R. C., Arizmendi, A. M., Eguiarte, L. E., \& Corcuera, P. M. (2012). Changes in composition, diversity and structure of woody plants in successional stages of tropical dry forest in southwest Mexico. Revista Mexicana de Biodiversidad, 83(4), 10961109. doi: 10.22201/ib.20078706e.2012.4.1011

Angiosperm Phylogeny Group [APG]. (2016). An update of the Angiosperm Phylogeny Group classification for the orders and families of flowering plants: APG IV. Botanical Journal of the Linnean Society, 181(1), 1-20. doi: 10.1111/boj.12385

Beltrán-Rodríguez, L., Valdez-Hernández, J. J., Luna-Cavazos, M., Romero-Manzanares, A., Pineda-Herrera, E., MaldonadoAlmanza, B., Borja de la Rosa, M. A. \& Blancas-Vázquez, J. (2018). Estructura y diversidad arbórea de bosques tropicales caducifolios secundarios en la Reserva de la Biosfera Sierra de Huautla, Morelos. Revista Mexicana de Biodiversidad, 89(1), 108-122. doi: 10.22201/ib.20078706e.2018.1.2004

Becknell, J. M., Kucek, L. K., \& Powers, J. S. (2012). Aboveground biomass in mature and secondary seasonally dry tropical forests: a literature review and global synthesis. Forest Ecology and Management, 276, 88-95. doi: 10.1016/j.foreco.2012.03.033

Bongers, F., Poorter, L., Hawthorne, W. D. \& Sheil, D. (2009). The intermediate disturbance hypothesis applies to tropical forests, but disturbance contributes little to tree diversity. Ecology Letters, 12(8), 1-8. doi: 10.1111/j.1461-0248.2009.01329.x

Brower, J. E., Zar, J. H., \& Ende, C. N. (1998). Field and laboratory methods for general ecology (4a. ed.). Boston, USA: WCB-McGraw-Hill.

Chazdon, R. L., Letcher, S. G., Van Breugel, M., Martínez-Ramos, M., Bongers, F., \& Finegan, B. (2007). Rates of change in tree communities of secondary Neotropical forests following major 
disturbances. Philosophical Transactions of the Royal Society B: Biological Sciences, 362(1478), 273-289. doi: 10.1098/rstb.2006.1990

Ceballos, G., Martínez, A. L., García, E., Creel, J. B., \& Dirzo, R. (2010). Diversidad, amenazas y áreas prioritarias para la conservación de las selvas secas del Pacifico de México. México: Conabio/Fondo de Cultura Económica.

Colwell, R. K. (2009). EstimateS v. 8.2.0. Statistical estimation of species richness and shared species from samples. USA: University Connecticut.

Connell, J. H. (1978). Diversity in tropical rain forests and coral reefs. Science, 199(4335), 1302-1310. doi: 10.1126/science.199.4335.1302

Cordero, J. (2003). Árboles de Centroamérica: un manual para extensionistas. UK: Bib. Orton IICA / CATIE, 2003.

Chao, A., Ma K. H., Hsieh, T. C., \& Chun-Huo, C. (2016). Species richness prediction and diversity estimation in $\mathrm{R}$ (SpadeR). Recuperado de https://chao.shinyapps.io/SpadeR/

Derroire, G., Balvanera, P., Castellanos-Castro, C., Decocq, G., Kennard, D. K., Lebrija-Trejos, E., Leiva, J., Oden, P., Powers, J. S., Rico-Gray, V., Tigabu, M., \& Healey, R. (2016). Resilience of tropical dry forests - a meta-analysis of changes in species diversity and composition during secondary succession. Oikos, 125(10), 1386-1397. doi: 10.1111/oik.03229

Dupuy, J. M., Hernández-Stefanoni, J. L., Hernández-Juárez, R. A., Tetetla-Rangel, E., López-Martínez, J. O., Leyequién-Abarca, E., Tun-Zul, F., \& May-Pat, F. (2012). Patterns and correlates of tropical dry forest structure and composition in a highly replicated chronosequence in Yucatan, Mexico. Biotropica, 44(2), 151-162 2012. doi: 10.1111/j.1744-7429.2011.00783.x

Espinosa-Jiménez, J. A., López-Cruz, A., Pérez-Farrera, M. A., \& López, S. (2014). Inventario florístico de la cañada la Chacona-Juan Crispín y zonas adyacentes, Depresión Central de Chiapas, México. Botanical Sciences, 92(2), 205-241. doi: 10.17129/botsci.30

Ewel, J. (1977). Differences between wet and dry successional tropical ecosystems. International Journal of Tropical Geology, Geography and Ecology, 1(2), 103-117.

García, E. (2004). Modificaciones al sistema de clasificación climática de Köppen. México: Instituto de Geografía y Universidad Nacional Autónoma de México.

Guariguata, M. \& Ostertag, R. (2001). Neotropical secondary forest succession: changes in structural and functional characteristics. Forest Ecology and Management, 148 (1-3), 185-2006. doi: 10.1016/S0378-1127(00)00535-1

Gardner, T.A., Barlow, J., R. Chazdon, Ewers, R. M., Harvey, C. A., Peres, C. A., \& Sodhi, N. S. (2009). Prospects for tropical forest biodiversity in a human-modified world. Ecology Letters, 12(2), 561582. doi: 10.1111/j.1461-0248.2009.01294.x

Gelviz-Gelvez, S. M. \& Pavón, H. N. (2013). Diversidad de especies arbustivas en una zona semiárida de México. Ciencias Forestales y Medio Ambiente, 19(3), 223-335. doi: 10.5154/r.rchscfa.2012.08.049

Instituto Nacional de Estadística y Geografía [Inegi]. (1985). Carta Edafológica 1:250 000 Tuxtla Gutiérrez. E15-11. México: Inegi.

Hammond, D.S. (1995). Post-dispersal seed and seedling mortality of tropical dry forest trees after shifting agriculture, Chiapas, Mexico. Journal of Tropical Ecology, 11(2), 295-313. doi: $10.1017 / \mathrm{s} 0266467400008762$

Hilje, B., Calvo-Alvarado, J., Jiménez-Rodríguez, C., \& SánchezAzofeifa, A. (2015). Tree species composition, breeding systems, and pollination and dispersal syndromes in three forest successional stages in a tropical dry forest in Mesoamerica. Tropical Conservation Science, $\quad 8(1), \quad 76-94 . \quad$ doi: 10.1177/194008291500800109

Jost, L. (2006). Entropy and diversity. Oikos 113(2), 110-116. doi: 10.1111/j.2006.0030-1299.14714.x

Kalacska, M., Sánchez-Azofeita, G.A., Calvo-Alvarado, J. C., Quesada, M., Rivardy, B. \& Janzen. D. H. (2004). Species composition, similarity and diversity in three successional stages of a seasonally dry tropical forest. Forest Ecology and Management, 200(1-3), 227247. doi: doi.org/10.1016/j.foreco.2004.07.001

Leirana-Alcocer, J. L., Hernández-Betancourt, S., Salinas-Peba, L. \& Guerrero-Gónzalez, L. (2009). Cambios en la estructura y composición de la vegetación relacionados con los años de abandono de tierras agropecuarias en la selva baja caducifolia espinosa de la reserva de Dzilam, Yucatán. Polibotánica, 27, 53-70.

Lebrija-Trejos, E., Meave, J.A., Poorter, L., Pérez-García, E.A., \& Bongers, F. (2010). Pathways, mechanisms and predictability of vegetation change during tropical dry forest succession. Perspective of Plant Ecology, 12(4), 267-275. doi: 10.1016/j.ppees.2010.09.002

Lebrija-Trejos, E., Bongers, F., Pérez-García, E.A., \& Meave, J.A. (2008). Successional change and resilience of a very dry tropical deciduous forest following shifting agriculture. Biotropica, 40(4), 422-431. doi: 10.1111/j.1744-7429.2008.00398.x

López, E. J. G. (2006). Estimación de tormentas y avenidas para el diseño de las obras de protección del Río Sabinal. Tesis de maestría, Universidad Autónoma de Chiapas, Chiapas, México.

López-Toledo, J.F., Váldez-Hernández, J.I., Pérez-Farrera, M.A., \& Celina-Alcalá, V.M. (2012). Composición y estructura arbórea de un bosque tropical estacionalmente seco en la reserva de la 
biosfera La Sepultura, Chiapas. Revista Mexicana de Ciencias Forestales, 3(12), 44-55. doi: 10.29298/rmcf.v3i12.507

López-Jiménez, L., Durán-García, R., \& Dupuy-Rda, J.M. (2019). Recuperación de la estructura, diversidad y composición de una selva mediana subperennifolia en Yucatán, México. Madera y Bosques, 25(1), 1-16. doi: 10.21829/myb.2019.2511587

Marin-Spiotta, E., Cusak, C., Ostertag, R., \& Silver, W.L. (2007). Trends in above and belowground carbon with forest regrowth after agricultural abandonment in the Neotropics. En W. M. Randall (Ed.), Post-agricultural sucession in the Neotropics (pp. 22-72). USA: Springer. doi: 10.1007/978-0-387-33642-8_2

Martínez-Yrizar, A, Sarukhan, J., Pérez-Jiménez, A., Rincon, E., Maass, J. M., Solis-Magallanes, A., \& Cervantes, L. (1992). Above-ground phytomass of a tropical deciduous forest on the coast of Jalisco, Mexico. Journal of Tropical Ecology, 8(1), 87-96. doi: 10.1017/S0266467400006131

Magurran, A. E. (2004). Measuring biological diversity. UK: Blackwell Plublishing.

Miranda, F. (1952). La vegetación de Chiapas: primera parte (1a ed.). México: Gobierno del Estado de Chiapas.

McArdle, B.H., \& Anderson, M.J. (2001). Fitting multivariate models to community data: a comment on distance-based redundancy analysis. Ecology, 82(1), 290-297. doi: 10.1890/00129658(2001)082[0290:FMMTCD]2.0.CO;2

Minchin, P.R. (1987). An evaluation of relative robustness of techniques for ecological ordinations. Vegetatio, 69(1/3), 89-107. doi: 10.1007/BF00038690

Mora, F., Martínez-Ramos, M., Ibarra-Manríquez, G., Pérez-Jiménez A., Trilleras, J., \& Balvanera, P. (2015). Testing chronosequences through dynamic approaches: time and site effects on tropical Dry Forest succession. Biotropica, 47(1), 38-48. doi: 10.1111/btp.12187

Mueller-Dombois, D., \& Ellemberg, H. (2002). Aims and methods of vegetation ecology (2nd. ed.). New Jersey, USA: Blackburn Press.

Murphy, P.G., \& Lugo, A.E. (1986). Structure and biomass of a subtropical dry forest in Puerto Rico. Biotropica, 18(2), 89-96. doi: $10.2307 / 2388750$

Norden, N., Angarita, A. H., Bongers, F., Martínez-Ramos, M., Granzow-De la Cerda, I., Breugel, M., Lebrija-Trejos, E., \& Chazdon, R. L. (2015). Succesional dynamic in Neotropical forest are as uncertain as they are predictable. PNAS, 112(26), 80138018, doi: 10.1073/pnas.1500403112

Pérez-Farrera, M.A., \& Espinosa, E. M. (2010). Depresión CentralComalapa, Chiapas. En Ceballos, G., Martínez, L., García, A.,
Espinoza, E., Creel, J. B., \& Dirzo, R. (Eds.). Diversidad, amenazas y áreas prioritarias para la conservación de las selvas secas del Pacífico de México (pp. 543-547). México: Conabio.

Poorter, L., Bongers, F., Aide, T. M., Almeyda-Zambrano, A. M., Balvanera, P., Becknell, J. M., Boukili, V., Brancalion, P. H. S., Broadbent, E. N., Chazdon, R. L., Craven, D., de AlmeidaCortez, J. S., Cabral, G. A. L., de Jong, B. H. J., Denslow, J. S., Dent, D. H., DeWalt, S. J., Dupuy, J. M., Durán, S. M., EspíritoSanto, M. M., Fandino, M. C., César, R. G., Hall, J. S., HernándezStefanoni, J. L., Jakovac, C. C., Junqueira, A. B., Kennard, D., Letchet, S. G., Licona, J. C., Lohbeck, M., Marín-Spiotta, E., Martínez-Ramos, M., Massoca, P., Meave, J. A., Mesquita, R., Mora, F., Muñoz, R., Muscarella, R., Nunes, Y. R. F., OchoaGaona, S., De Oliveira, A. A., Orihuela-Belmonte, E., PañaClaros, M., Pérez-García, E. A., Piotto, D., Powers, J. S., Rodríguez-Velázquez, J., Romero-Pérez, I. E., Ruíz, J., Saldarriaga, J. G., Sánchez-Azofeifa, A., Schwartz, N. B., Steininger, M. K., Swenson, N. G., Toledo, M., Uriarte, M., Van Breugel, M., Van der Wal, H., Veloso, M. D. M., Vester, H. F. M., Vicentini, A., Viera, I. C. G., Vizcarra-Bentos, T., Williamson, G. B., \& Rozendaal, D. M. A. (2016). Biomass resilience of Neotropical secondary forests. Nature, 530(7589), 211-214. doi: $10.1038 /$ nature16512

Quesada, M., Sanchez-Azofeifa, G.A., Álvarez-Anorve, M., Stoner, K.E., Avila-Cabadilla, L., Calvo-Alvarado, J., Castillo, A., EspíritoSanto, M.M., Fagundes, M., Fernandes, G.W., Gamon, J., Lopezaraiza-Mikel, M., Lawrence, D., Cerdeira, M. L., Power, J. S., Neves, F., Rosas-Guerrero, V., Sayago, R., \& SánchezMontoya, G. (2009). Succession and management of tropical dry forest in the Americas: review and new perspectives. Forest Ecology Management, 258(6), 1014-1024. doi: 10.1016/j.foreco.2009.06.023

R Core Team. (2013). R: a language and environment for statistical computing. Recuperado de http://www.R-project.org/

Read, L. \& Lawrence, D. (2003). Recovery of biomass following shifting cultivation in dry tropical forest of the Yucatan. Ecological Applications, 13(1): 85-97. doi: 10.1890/10510761(2003)013[0085:ROBFSC]2.0.CO;2

Reyes-Garcia, A., \& Sousa, M. (1997). Depresión Central de Chiapas, la selva baja caducifolia. Listados Florísticos de México XVII. México: Universidad Nacional Autónoma de México/Instituto de Biología.

Rocha-Loredo, A.C., Ramírez-Marcial, N., \& González-Espinosa M. (2010). Riqueza y diversidad de árboles del bosque tropical caducifolio en la Depresión Central de Chiapas. Boletín de la Sociedad Botánica de México, 87, 89-103. doi: 10.17129/botsci.313

Rozendaal, M. A., Chazdon, R. L., Arreola-Villa, F., Balvanera, P., Bentos, T. V., Dupuy, J. M., Hernández-Stefanoni, L., Jakova, C. 
C., Lebrija-Trejos, E., Lohbeck, M., Martínez-Ramos, M., Massoca, P., Meave, J. A., Mesquita, R., Mora, F., Pérez-García, E. Romero-Pérez, E., Saenz-Pedroza, I., Breugel, M., William, B., \& Bongers, F. (2016). Demographic drivers of aboveground biomass dynamics during secondary succession in Neotropical dry and wet forests. Ecosytems, 20(2), 340-353. doi: 10.1007/s10021-016-0029-4

Rüger, N., Williams-Linera, G., Kissling, W.D., \& Huth, A. (2008). LongTerm impacts of fuelwood extraction on a tropical montane cloud forest. Ecosystems, 11, 868-881. doi: 10.1007/s10021-008-9166-8

Rzedowski, J. (1991). El endemismo en la flora fanerogámica mexicana: una apreciación analítica preliminar. Acta Botánica Mexicana, 15, 47-64. doi: 10.21829/abm15.1991.620

Rzedowski J., \& Calderon, G. (2013). Datos para la apreciación de la flora fanerogámica del bosque tropical caducifolio de México. Acta Botánica Mexicana, 102, 1-23. doi: 10.21829/abm102.2013.229

Sánchez-Molina, D. (2014). Estructura y composición florística de la subcuenca del rio Sabinal, Chiapas, México. Tesis de licenciatura, Universidad de Ciencias y Artes de Chiapas, Chiapas, México.

Secretaria de Medio Ambiente y Vivienda [Semavi]. (2009). Programa de ordenamiento ecológico territorial de la subcuenca del Río Sabinal. México: Semavi.

Sheil, D., \& Burslem, D.F. (2003). Disturbing hypotheses in tropical forests. Trends Ecology Evolution 18(1), 18-26. doi: 10.1016/S01695347(02)00005-8

The International Plant List [INPI]. (2012). The international plant names index. Recuperado de https://www.ipni.org/

Trejo, I. (1998). Distribución y diversidad de selvas bajas de México: relaciones con el clima y el suelo. Tesis de doctorado, Universidad Autónoma de México, D.F., México.

Trejo I., \& Dirzo, R. (2000). Deforestation and seasonally dry tropical forest: a national and local analysis in Mexico. Biological Conservation, 94(2), 133-142. doi: 10.1016/S0006-3207(99)00188-3

Vargas, M.F., Escobar, S., \& Del Ángel, R. (2000). Áreas naturales protegidas de México con decretos federales. Recuperado de http://www.paot.mx/centro/ine-semarnat/anp/AN01.pdf
Vargas, G., Werden, L. K., \& Powers, J.S. (2015). Explaining legume success in tropical dry forests based on seed germination niches: a new hypothesis. Biotropica, 47(3), 277-280. doi: 10.1111/btp.12210

Vázquez-Yanes, C., Batis, A. I., Alcocer, S. M., Gual, M. D., \& Sánchez, C. D. (1999). Árboles y arbustos nativos potencialmente valiosos para la restauración ecológica y la reforestación, Informe final- J084. México: Conabio/Instituto de Ecología/UNAM.

Vieira, D. L.M., \& Scariot, A. (2006). Principles of natural regeneration of tropical dry forests for restoration. Restoration Ecology, 14(1), 1120. doi: 10.1111/j.1526-100X.2006.00100.x

Villalobos, S.M. (2012). Patrones, procesos y mecanismos de la comunidad regenerativa de un bosque tropical caducifolio en un gradiente sucesional. Tesis de doctorado, Universidad Nacional Autónoma de México, Morelia, México.

William-Linera, G., Álvarez-Aquino, C., Hernández-Ascención, E., \& Toledo, M. (2011). Early successional sites and the recovery of vegetation structure and tree species of the tropical dry forest in Veracruz, Mexico. New Forests, 42(1), 131-148. doi: $10.1007 /$ s11056-010-9242-8

World Agroforestry [Icraf] (s/f). Tree functional attribute and ecological database Recuperado de http://db.worldagroforestry.org

Manuscrito recibido el 08 de octubre de 2019

Aceptado el 30 de abil de 2020

Publicado el 17 de diciembre de 2020

Este documento se debe citar como:

Gordillo R., M. C., Pérez F., M. A., \& Castillo S., M. A. (2020). Estructura y composición arbórea del bosque tropical caducifolio secundario en la Depresión Central, Chiapas, México. Madera y Bosques, 26(3), e2632055. doi: 10.21829/myb.2020.2632055

Madera y Bosques por Instituto de Ecología, A.C. se distribuye bajo una Licencia Creative Commons Atribución-NoComercialCompartirlgual 4.0 Internacional. 